\title{
EPR and luminescence data on the nitrogen aggregation in diamonds from Snap Lake dyke system
}

\author{
V.A. Nadolinny ${ }^{1}$, O.P. Yurjeva ${ }^{1}$, N.P. Pokhilenko ${ }^{2}$ \\ ${ }^{1}$ Institute of Inorganic Chemistry SB RAS, Novosibirsk, Russia, \\ ${ }^{2}$ Institute of Geology and Mineralogy SB RAS, Novosibirsk, Russia
}

In this communication we describe more 200 samples of diamonds from the vast deposit on the American continent, discovered in the 90 s in the region of Snap Lake, province Slave, Canada. The samples have no inclusions and looked greenish to brown tinted. Their properties were studied in details using optical and EPR spectroscopy. Two main groups of diamonds were separated: the first of them are related to IaB type with maximum degree of defects aggregation: N3, B nitrogen defects and B' clusters of interstitials, and the second part of specimens has one type emission under visual examination of photoluminescence: green shell and blue core. As a rule these diamonds are the IaA type samples with nitrogen mainly in A-form. 1 1aB diamonds with the traces of plastic
deformation.

The $1 \mathrm{aB}$ diamonds studied are 60 crystals. Preliminary IR spectral examination was carried out to select the crystals with B and B' centers. The diamonds from the Canadian deposit contain $30 \%$ such crystals.

EPR spectra were obtained with an E109 Varian EPR spectrometer operating at X- band at 300 and $77 \mathrm{~K}$. IR spectra were taken by a FT-IR Paragon 1000 with resolution $1 \mathrm{~cm}^{-1}$ in the range $450-4400 \mathrm{~cm}^{-1}$ and a FIR Bruker spectrometer. The luminescence spectra were obtained with a spectrometer DFS-24 at $77 \mathrm{~K}$. The crystals were annealed using a high pressure apparatus of split sphere type.

In crystal No. SL-00/106, IR $1175 \mathrm{~cm}^{-1}$ and $1360 \mathrm{~cm}^{-1}$ bands dominate, and also a small portion of nitrogen in A-centers (30-50ppm) is observed. Decomposing IR spectra of these crystals shows a small discrepancy in $1332 \mathrm{~cm}^{-1}$ intensity from B-centers. Recent IR investigations have shown that the $\mathrm{N}^{+}$nitrogen state has also absorption at $1332 \mathrm{~cm}^{-1}$.

The $1 \mathrm{aB}$ diamonds examined have strong blue or whitish-blue glow. Their PL spectra exhibit an intense system at $415 \mathrm{~nm}$ (see Fig.1, top), but the peak intensity of the zero-phonon line (ZPL) appeared reduced as compared to the intensity of the side-band This distinguishes the system shown from that known in the literature and no self-absorption in the ZPL is likely. A well-defined phonon structure is easily observable indicating a consistency of the defect structure and determining its symmetry. The N3 system is accompanied by a group of weak narrow zero phonon lines at 407.8, 409 and $411 \mathrm{~nm}$ : 409 was always observed and sometimes additional lines at the other two wavelengths.

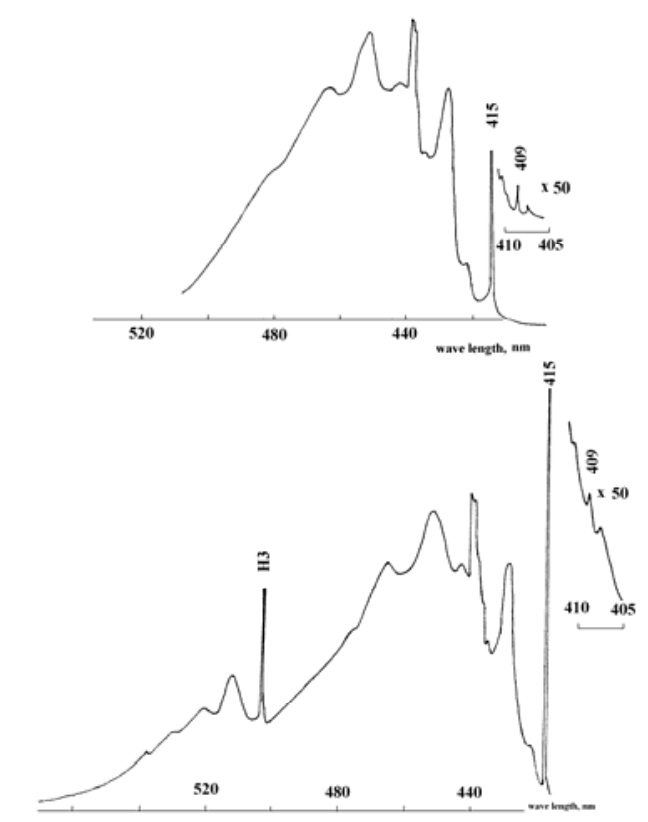

Fig. 1. PL spectra of $1 \mathrm{aB}$ diamond before (top) and after (bottom) HPHT treatment.

At room temperature, the EPR spectra of the crystals studied consist of only a broad line with $\mathrm{g}=2,0031$ and $\Delta \mathrm{H}_{1 / 2}=11.5$ Gauss (see Fig.2, top), which is due to P2 center (Nadolinny et al, 2004). The diamonds were optically illuminated in the EPR cavity at 77 and 300 $\mathrm{K}$. At room temperature, illumination of the crystals has no influence on the P2 EPR spectrum. Cooling the sample to $77 \mathrm{~K}$ causes the broad P2 line to disappear. Light excitation with $\lambda<380 \mathrm{~nm}$ at $77 \mathrm{~K}$ causes the broad P2 line to restore, and an additional P1 EPR spectrum and a single narrow line with $g=2.0027$ and $\Delta \mathrm{H}_{1 / 2}=1$ Gauss to appear. The line width of the P1 spectrum is estimated as $\Delta \mathrm{H}_{1 / 2}=2.5$ Gauss. The estimation indicates a P1 content in the crystal SL00/106 of $6 \mathrm{ppm}$, which is not typical of IaB diamonds.

The dynamics of the photo-excited P2 EPR spectrum was studied. UV excitation showed slow decreasing EPR spectra in the dark. Light of $\lambda>400 \mathrm{~nm}$ at $77 \mathrm{~K}$ produces a decrease of the P2 EPR spectrum to the level observed after crystal cooling. Under these conditions the P1 EPR spectrum disappeared and the 
narrow line was restored to the intensity it had in the dark after crystal cooling. All changes observed are reversible on UV excitation followed by red illumination. The spectrum left after the red illumination consists of poorly resolved P2 EPR lines. The broad line can be simulated by increasing the line width of the resolved P2 EPR spectrum to 2.5 Gauss.

HPHT annealing the diamonds studied was carried out in two stages: 1) at $7 \mathrm{GPa}, 1850^{\circ} \mathrm{C}$ for $12 \mathrm{~h}$ and 2) at 7 $\mathrm{GPa}, 2000^{\circ} \mathrm{C}$ for $12 \mathrm{~h}$. The first annealing stage reduced photo-excitation effect on the EPR spectra and a weak P1 signal was detectable at room temperature in the absence of excitation. The second annealing at $2000^{\circ} \mathrm{C}$ causes the broad P2 line to disappear and a single narrow EPR line and simultaneously an intense P1 center and P2 center with resolved HFS to become observable (see Fig.2, bottom). The line width of an individual line for the both centers is 0.46 Gauss.

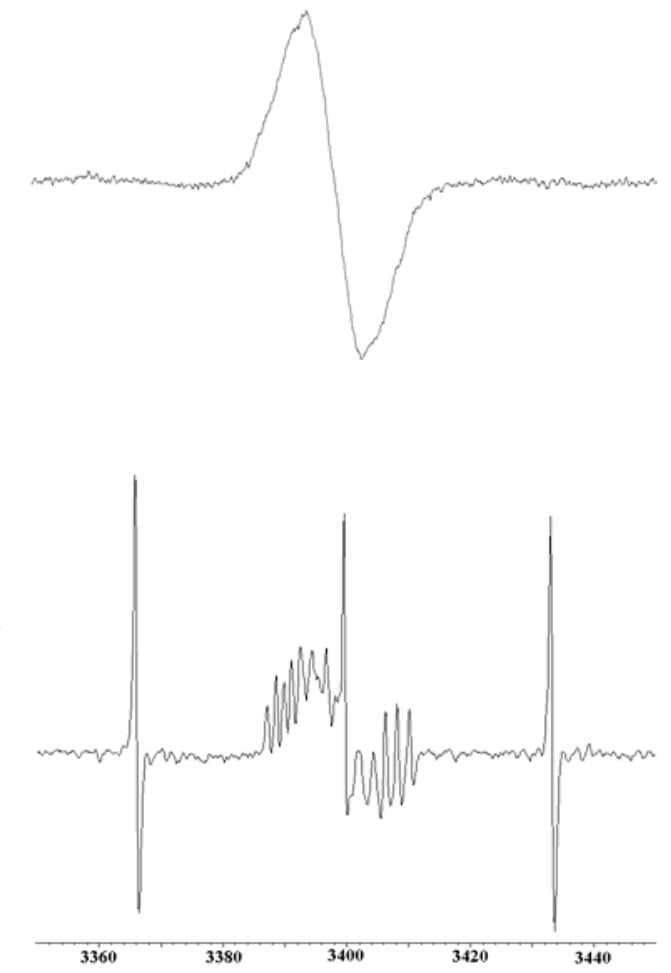

Fig.2. EPR spectra of $1 \mathrm{aB}$ diamond before (top) and after (bottom) HPHT treatment. Abscissa axis is magnetic field in Gauss

In PL spectra the second annealing stage results in some increase in intensity of the blue band as a whole and removal of the broadening effect of $415 \mathrm{~nm} \mathrm{ZPL}$, its peak intensity being enhanced markedly (Fig. 1, bottom). Simultaneously there were observed a decreased $409 \mathrm{~nm}$ line and an appearance of a rather intense H3 system (see Fig. 1, bottom). The latter is likely to be responsible for the greenish color of the annealed crystal.

Very dramatic changes can be seen in the IR spectra after the second annealing stage, the intensities of all optical systems were found to be decreased by a factor of $2-3$.

\section{1aA type diamonds with the traces of a plastic deformation}

To investigate HTHP treatment in diamonds with traces of plastic deformation a set of brown crystals from the Canadian deposit was selected. Their IR spectra present 1282, 1175 and $1130 \mathrm{~cm}^{-1}$ bands. EPR spectra show the P1 center, the separated W7 nitrogen pairs (Scherbakova et al, 1975) and a single line with $g$ $=2.0027$ and $\Delta \mathrm{H}=1$ Gauss from dangling bonds in the core of dislocations. The crystals selected show a rather intense green glow, the $490.7 \mathrm{~nm}$ system (phonon energies of $68,93,114,158,165 \mathrm{meV}$ ) being dominant in PL spectra. As a rule, it was accompanied by the H3 and $\mathrm{H} 4$ systems of a different strength. A detailed examination of the blue spectral region allows us to define weak systems at 406 and $423 \mathrm{~nm}$. In the cases where these systems were not masked by the usually more intense $415 \mathrm{~nm}$ center their phonon structure was carefully analyzed (406 nm - 46, 82, 93, $160 \mathrm{meV} ; 423$ $\mathrm{nm}-40,65,93,128,165 \mathrm{meV}$ ) ( see Fig. 3).

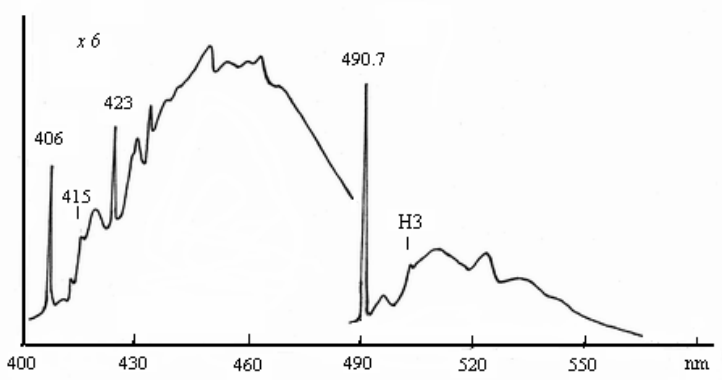

Fig.3. PL spectrum of 1aA crystal with $490.7 \mathrm{~nm}$ system.

Our observations show that the 423 and $406 \mathrm{~nm}$ systems always accompany $490.7 \mathrm{~nm}$, however no correlation between their intensities was established. Investigations of the optical and EPR spectra in parallel have shown that the $490.7 \mathrm{~nm}$ center has an analogue in the EPR single narrow line with $g=2.0027$.

$\mathrm{P}, \mathrm{T}$ treatment of these $1 \mathrm{aA}$ diamonds was performed at $7 \mathrm{GPa}$ at $1850^{\circ} \mathrm{C}$ for $12 \mathrm{~h}$. In the crystals treated, the W7 center and the single line from dangling bonds in the EPR spectra and also the 490.7, 406, $423 \mathrm{~nm}$ and H4 optical systems disappeared. The color of P,T treated diamonds changed to green. The intensity of the green glow was enhanced considerably (in some cases by more than two orders). The strongly broadened 503 ZPL is noteworthy.

The existence of an ionized nitrogen pair in the structure of the W7 center indicates electron transfer from this center to an electron acceptor. In these crystals possible acceptors are dangling bonds in the core of dislocations. The creation of such donoracceptor pairs with different distances between components implies the existence of a continuous system of electronic states with large dipole moments. This allows us to postulate that the brown color of the 
diamonds under study is due to bands of charge transfer in such donor-acceptor pairs. The result of interaction between the main form of nitrogen defects in these crystals (A-centers) and dislocations is the disruption of $\mathrm{A}$ centers by shifting carbon layers and creation of such centers as W7, H3.

\section{Discussion}

laB type diamonds contain the nitrogen impurity mainly in B and P2 forms. These centers are forms of high nitrogen aggregation in the diamond. An unusually high P1 content for this type of diamonds is the surprising thing in the diamonds we have studied, as well as that all $\mathrm{P} 1$ centers are in the ionized state $\mathrm{N}^{+}$. The ratio obtained between $\mathrm{P} 1, \mathrm{~A}-$ and $\mathrm{B}$-centers doesn't agree with the kinetics of the transformation of nitrogen defects. The production of $\mathrm{P} 1$ centers in this type of the diamond suggests the possibility of disruption of complicated nitrogen defects. Dissociation of the complex nitrogen defects on annealing higher than $2300^{\circ} \mathrm{C}$ is a well-established experimental fact, but our effects occur in temperature range $1850-2000^{\circ} \mathrm{C}$. Under these conditions a known interaction between interstitial carbon atoms and nitrogen defect occurs where the interstitial carbon atom substitutes nitrogen in the regular lattice position, but this mechanism does not account for all effects observable in EPR and optics. We postulate that a plastic deformation of the diamonds can be such a possible mechanism for disruption of complicated nitrogen defects.

During plastic deformation, as is well known for the W7 center in laA diamonds (Scherbakova et al, 1975), one of nitrogen atoms in the B-center structure seems shifted by sliding carbon layers along a $\{111\}$ plane and in a [110] direction. The observed P1 and P2 centers show quite a large separation between these centers. In addition to the possible displacement of P1 and P2 centers located on opposite sides of the sliding plane, at high temperature they may also diffuse along the directions of the stress gradient away from one another along the sliding plane. In other words, under these conditions the P1 and P2 centers don't revert back to B-centers. This mechanism can account for a different $\mathrm{P} 2$ content in the $1 \mathrm{aB}$ natural diamonds. The appearance of the $\mathrm{N}^{+}$nitrogen charge state in these crystals is due to close location of dislocations whose dangling bonds in the core are electron acceptors. The observed disappearance of the broad P2 EPR line with a decrease in temperature to $77 \mathrm{~K}$ is associated with the charge-transfer process between $\mathrm{P} 2$ centers and DB of dislocation on the assumption that the location of the energy level for one of these two centers changes. Because the structure and symmetry of the P2 center is seen to be invariable, as shown by lack of change in the optical spectrum, such an effect may be due to a change in the energy level at the dislocation. Usually as the temperature decreased the energy level is lowered. In our case this means that on decreasing the temperature the electron transfer from P2 centers to DBs of dislocations occurs creating diamagnetic $(\mathrm{P} 2)^{+}$ and DB states. At low temperature UV light excites

the electrons from DB to the conduction band and then they are captured by $\mathrm{N}^{+}$and $(\mathrm{P} 2)^{+}$electron traps. This allows the P1 and P2 centers to be observed in EPR spectra. Red illumination released the electron traps, turning the electrons back to the DB levels and $\mathrm{P} 1$ and $\mathrm{P} 2$ centers return to diamagnetic $\mathrm{N}^{+}$and $(\mathrm{P} 2)^{+}$ states. That these effects are absent after annealing out the dislocations supports this model.

Removing dislocations from the crystal allows both these centers to be observed at room temperature without any additional excitation. The P1 and P2 EPR line widths are evidences that a broadening of the EPR lines in the crystals examined is due to the lattice stress in vicinity of the dislocation core. The same reason may explain the ZPL behavior of the $415 \mathrm{~nm}$ PL system. The major portion of the P2 centers observed are assumed to be located close to the dislocation core. The $409 \mathrm{~nm}$ PL system is due to the negative charge state of the P2 center and increasing intensity of the $415 \mathrm{~nm}$ and $409 \mathrm{~nm}$ PL bands on red illumination corresponds to the process of electron removal from P1 centers and its capture by $(\mathrm{P} 2)^{+}$.

All experimental data obtained here support the hypothesis of the disruption of the B-center by the plastic deformation. It is very interesting, that the $1 \mathrm{aB}$ crystals studied have no brown color. Apparently the brown color is an inherent characteristic of the $1 \mathrm{aA}$ diamonds with traces of plastic deformation. Because the nitrogen centers are donors of electrons, it seems likely the brown color is due to the charge transfer band from nitrogen centers to DB in the dislocation core. For 1aA diamonds an energy level of the A center is positioned higher than that for $\mathrm{DB}$ and here the electron transfer is effected from the A center and its derivative defects to $\mathrm{DB}$. For the $1 \mathrm{aB}$ diamonds the energy levels in the P2 and B-centers are located lower than those for $\mathrm{DB}$ and the color of the $1 \mathrm{aB}$ crystals depends on $\mathrm{P} 1$ and $\mathrm{A}$-center contents.

The decrease in the content of A, B and B' defects in the $\mathrm{P}, \mathrm{T}$ treated $1 \mathrm{aB}$ crystals with a trace of plastic deformation is a very interesting and very important result. It means that on removing dislocations they disrupt the complicated forms of nitrogen aggregation to make the simpler defects such as P1, P2, H3. These results may explain the failure of many experiments attempting to change the color of diamonds with the traces of plastic deformation by $\mathrm{P}, \mathrm{T}$ treatment.

\section{References}

Nadolinny V.A., Yuryeva O.P., Yellisseyev A.P., Pokhilenko N.P., Chepurov A.I., 2004. Disruption of nitrogen $\mathrm{B} 1$ centers in natural $1 \mathrm{aB}$ type diamonds at the plastic deformation and the behavior of the defects created on HTHP treatment. Dokladi Akademii Nauk, 399, 532-536.

Shcherbakova M.Ya., Sobolev E.V., Nadolinny V.A., Aksenov V.K., 1975. Dinitrogen centers in brown plastically deformed diamonds Dokladi. Akademii Nauk SSSR, 225, 566-569. 